\title{
Outage and Throughput of WPCN-SWIPT Networks with Nonlinear EH Model in Nakagami-m Fading
}

\author{
Ruihong Jiang ${ }^{1,2}$ (D), Ke Xiong 1,2,* (), Yu Zhang ${ }^{3,4}$, Li Zhou ${ }^{5, *}$, Tong Liu ${ }^{6}$ \\ and Zhangdui Zhong ${ }^{7}$ \\ 1 School of Computer and Information Technology, Beijing Jiaotong University, Beijing 100044, China; \\ rhjiang@bjtu.edu.cn \\ 2 Beijing Key Laboratory of Traffic Data Analysis and Mining, Beijing Jiaotong University, \\ Beijing 100044, China \\ 3 State Grid Energy Research Institute Co., Ltd., Beijing 102209, China; zhangyu2@sgeri.sgcc.com.cn \\ 4 School of Computer and Communication Engineering, University of Science and Technology, \\ Beijing 100083, China \\ 5 School of Information, Beijing Wuzi University, Beijing 101149, China \\ 6 Beijing Computing Center, Beike Industry Park, Beijing 100094, China; Liutong@bcc.ac.cn \\ 7 State Key Lab of Rail Traffic Control and Safety, Beijing Jiaotong University, Beijing 100044, China; \\ Zhdzhong@bjtu.edu.cn \\ * Correspondence: kxiong@bjtu.edu.cn (K.X.); zhoulibit@126.com (L.Z.)
}

Received: 24 December 2018; Accepted: 28 January 2019; Published: 29 January 2019

\begin{abstract}
This paper analyzes outage probability and reliable throughput performance of a multi-user wireless-powered communication network-simultaneous wireless information and power transfer (WPCN-SWIPT) network with the logistic function-based (LG) nonlinear energy-harvesting (EH) model in Nakagami- $m$ fading. Power-splitting (PS) receiver architecture is considered. The closed-form expressions of the system outage probability and the system reliable throughput are derived, and then the corresponding asymptotic expressions are also provided to achieve simpler calculation in the high-transmit power scenarios. Simulation results demonstrate the correctness of our derived analytical results and show that the systems under the LG nonlinear and linear EH models have very different performance behaviors. Moreover, since the LG nonlinear EH model is closer to the features of practical EH circuit than the linear one, using the LG nonlinear EH model can avoid the false output results of the system performance evaluation.
\end{abstract}

Keywords: WPCN; SWIPT; PS; Nakagami- $m$ fading; nonlinear EH; outage probability; reliable throughput

\section{Introduction}

\subsection{Background}

Recently, the exponential growth of wireless devices (WDs) and the requirement of the communication services are causing scarcity of energy. Moreover, most WDs, such as smart electronic terminals and wireless sensors, are powered by capacity-limited batteries, which restricts the working lifetime of energy-constrained networks, e.g., wireless sensor networks (WSN), Internet of Things (IoT), and wireless personal area networks (WPAN) [1-5]. To provide sustainable energy to WDs in energy-constrained networks, energy-harvesting (EH) technology has emerged as a promising solution [6-8]. In the EH family, solar, wind, thermal, and vibration energy are very popular renewable energy resources. However, because of their unpredictability and uncontrollability, it is difficult for them to support sustainable and reliable communications [9-11]. Moreover, there is another kind of 
energy source, i.e., radio-frequency (RF) energy, which delivers energy via RF signals. Since RF signals are controllable and independent of external conditions including weather and climate, RF-based EH is considered to be a promising solution to provide stable energy for low-power energy-constrained networks [12,13].

At present, there are two main application paradigms of RF-based EH technologies, i.e., simultaneous wireless information and power transfer (SWIPT) [14,15] and wireless-powered communication networks (WPCN) [16]. For SWIPT, it realizes wireless information transfer (WIT) and wireless power transfer (WPT) simultaneously with the same RF signals, which fully uses the features of wireless RF signals. Due to the large difference between information decoding (ID) and EH sensitives, i.e., $-60 \mathrm{dBm}$ for ID and $-10 \mathrm{dBm}$ for $\mathrm{EH}$, two practical receiver architectures, i.e., power-splitting (PS) and time switching (TS) receiver architectures, for implementing SWIPT were proposed in [17]. For WPCN, WDs first harvest energy over downlink WPT, and then use the harvested energy to perform uplink WIT, where ID and EH are performed by separated ID and EH receivers.

\subsection{Related Work}

So far, both SWIPT and WPCN have been widely studied, where one of the branches focused on analyzing the outage performance of them in various communication networks, see e.g., [18-22]. In [18], the outage probability and the ergodic capacity were investigated in amplify-and-forward (AF) relaying SWIPT systems. In [19], the system outage probability was minimized for two-way decode-and-forward (DF) relay SWIPT networks. In [20], the outage probabilities of both primary and secondary users were investigated for cooperative cognitive radio SWIPT networks. In [21], the system outage probability was minimized in multi-relay WPCN networks with DF relaying protocol and relay selection. In [22], the secrecy outage probability and the achievable information rate were explored in multi-user wiretap WPCN networks with an EH jammer and multiple eavesdroppers.

However, in these works mentioned above, only traditional linear EH model was adopted, where it was assumed that the amount of the harvested energy at WDs could be linearly increased with the input RF power (i.e., the received RF power at WDs). Recent works [23-25] showed that practical EH circuits generally is with a nonlinear feature due to their nonlinear components such as diodes, resistors, capacitors, etc. Therefore, using the traditional linear EH model cannot accurately characterize the real amount of energy that could be harvested at WDs. Thus, by fitting practical measurement data, a logistic function-based (LG) nonlinear EH model was proposed in [23]. As the LG nonlinear EH model is much closer to practical systems, it attracts increasing attention and has been studied in various wireless networks, see e.g., point-to-point networks [26,27], MISO networks [28,29], MIMO networks [30,31], NOMA networks [32], cognitive radio networks [33], and relay networks [34], where most of them adopted the LG nonlinear EH model to optimally allocate the system resources or design the SWIPT receiver's parameters.

Since the LG nonlinear EH model is too complex to track, especially for the system outage performance analysis, a piecewise (PW) nonlinear EH model was presented to approximate the LG one in [35], with which the system outage performance was investigated for wireless-powered dual-hop relaying MIMO network in independent Rayleigh fading. Due to its traceability, in [36], it was adopted to analyze and minimize the system outage probability of AF relaying SWIPT networks over Gaussian channels. In [37], the PW nonlinear EH model was employed to study the secure outage probability performance for wireless-powered multi-antenna DF relaying system in independent Rayleigh fading. Yet, in these works, only Rayleigh and Gaussian fading channels were investigated rather than Nakagami- $m$ fading channel.

On the other hand, since Nakagami- $m$ fading channel is more general and practical compared with Rayleigh and Gaussian channel fading models [38], some recent studies on SWIPT and WPCN began to discuss the system outage performance in Nakagami- $m$ fading, see e.g., [39-41]. In [39], the system outage capacity was studied for wireless-powered DF relaying networks in Nakagami- $m$ fading with the PW nonlinear EH model. In [40], the system outage probability and throughput were 
analyzed for hybrid SWIPT AF relaying system in Nakagami- $m$ fading with an approximate fractional (FR) nonlinear EH model (The fractional (FR) nonlinear EH model was presented in [41], which was $P_{\text {eh }}\left(P_{\text {in }}\right)=\frac{p_{1} P_{\text {in }}+p_{2}}{P_{\text {in }}+p_{3}}-\frac{p_{2}}{p_{3}}$, where $p_{1}, p_{2}$ and $p_{3}$ are constants determined by standard curve-fitting and depend on the type of $\mathrm{EH}$ receiver). In [42], the average outage probability and throughput of the system were investigated for multi-antenna WPCN networks with the LG nonlinear EH model in Nakagami- $m$ fading.

\subsection{Motivation}

In this paper, we also focus on the outage performance of RF-EH networks in Nakagami- $m$ fading, where the LG nonlinear EH is employed. The main different from existing works are presented as follows.

Firstly, although SWIPT and WPCN have been studied in the literature in the past few years, see e.g., [18-42], they were separately discussed. So far, few works have investigated them in a single system. As mentioned above, in WPCN systems, WPT is performed in downlink transmission and in the uplink transmission WIT is executed. Combined with the characteristics of SWIPT, WIT can be replaced by SWIPT when SWIPT is just used for information transfer. Moreover, SWIPT can also be used for EH, similar to WPT. Therefore, with WPCN and SWIPT, a WPCN-SWIPT (or WPT-SWIPT) system is considered in our paper.

Secondly, most existing works, see e.g., [35-37,41], investigated the system outage and throughput performance over Gaussian channel, Rayleigh fading channel, and Rician fading channel. Since Nakagami- $m$ fading is a generalized model, which is more accuracy and flexibility in matching the various empirically obtained measurement data than other models [38-41], we use it in our work.

Thirdly, in most existing works related to WPCN and SWIPT networks, see e.g., [18-22], the traditional linear EH model was used. To avoid the inaccurate analysis caused by the linear EH model, the LG nonlinear EH model is employed in our work.

\subsection{Contributions}

In this paper, we consider a multi-user WPCN-SWIPT system, where a power-free source node first harvests energy from a power station, and then uses the harvested energy to simultaneously transmit information and power to multiple energy-constrained terminal users. PS receiver architecture (since PS receiver architecture outperforms TS one with a larger rate-energy region [17], we consider PS architecture in our work) is used at each terminal user. The main contributions of our work are summarized as follows.

- A WPT-SWIPT protocol is presented including two phases: WPT phase and SWIPT phase. In the WPT phase, it can charge the power-free source node. In the SWIPT phase, the source node can transmit information and power to the terminal users simultaneously by using the harvested energy.

- The closed-form expressions of the system outage probability are derived. To make the result more concise, asymptotic expressions of the system outage probability are also presented in high-transmit power scenarios. Then, the expressions of the system reliable throughput are also provided. For comparison, the system performance with the linear EH model is also analyzed.

- Simulation results demonstrate the correctness of our derived analytical results and show that the systems under the LG nonlinear and linear EH models have very different performance behaviors. Using the LG nonlinear EH model can avoid false results of the system performance evaluation because the LG nonlinear EH model is closer to practice than the linear one.

The rest of this paper is organized as follows. Section 2 describes the system model. The outage probability and throughout of the system are analyzed in Section 3. Some numerical results are presented in Section 4 and finally, Section 5 concludes this paper. 


\section{System Model}

A multi-user WPCN-SWIPT system (From the perspective of MPS, it is a WPCN system where $\mathrm{S}$ first harvests energy from MPS in the downlink transmission, and uses the harvested energy to transfer information to $\mathrm{U}_{k}$ in the uplink transmission. From the perspective of $\mathrm{U}_{k}$, it is a SWIPT system where $S$ simultaneously transmits information and power to the PS-enabled $\mathrm{U}_{k}$ ) is considered to be shown in Figure 1, where a power-free source node (denoted as S) first harvests energy from a multi-antenna power station (denoted as MPS), and then consumes the harvested energy to transmit information and power to $K$ energy-constrained terminal users simultaneously. The $k$-th user is denoted as $\mathrm{U}_{k}$, where $k=1, \ldots, K$. Due to the barriers, there is no direct link from MPS to $\mathrm{U}_{k}$. Therefore, the energy-constrained terminal users need to harvest energy from $S$ to maintain their normal operations sometimes. MPS is equipped with $N_{\mathrm{t}} \geq 1$ antennas. $\mathrm{S}$ is equipped with single antenna. $\mathrm{U}_{k}$ is equipped with $N_{k} \geq 1$ antennas, which adopts PS receiver architecture to implement ID and EH function by employing SWIPT.

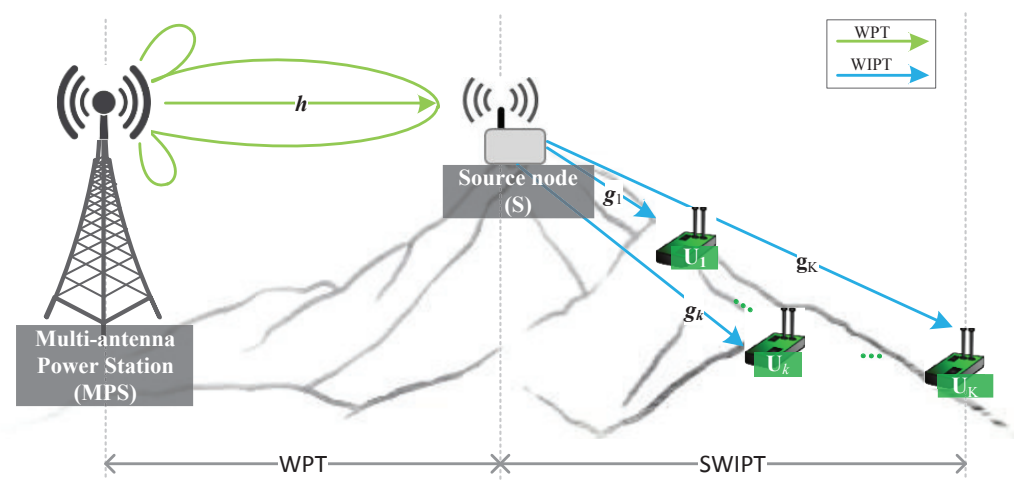

Figure 1. System model.

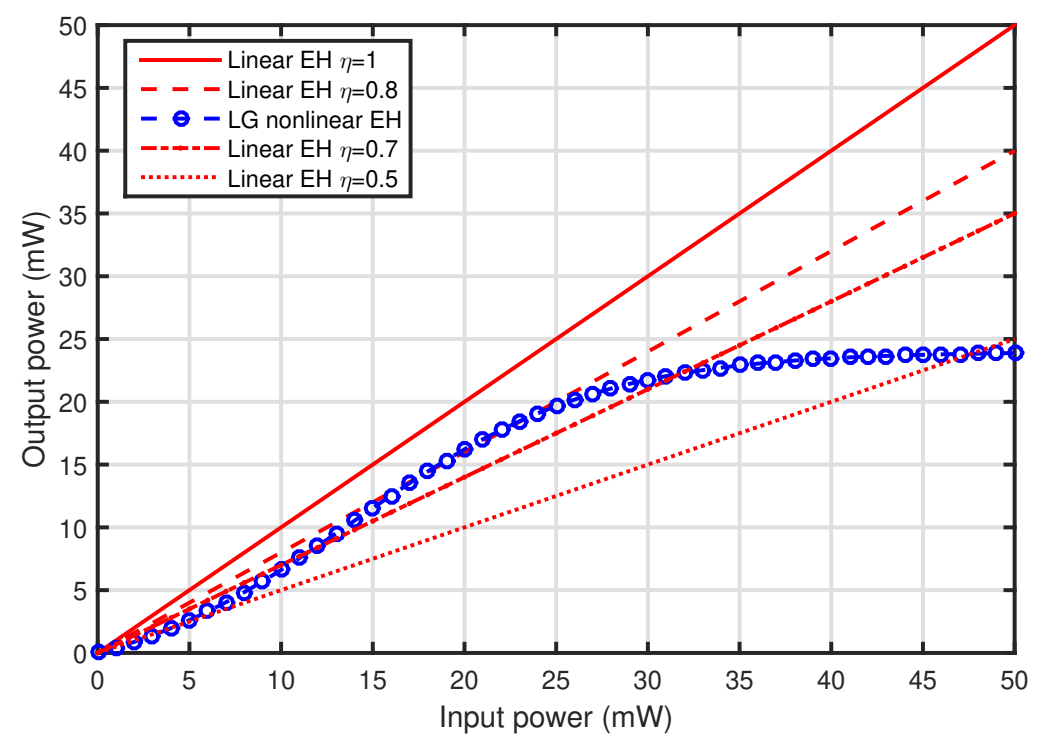

Figure 2. Nonlinear EH model vs. linear EH model.

\subsection{Channel Model}

For the system, both the large-scale path loss and the small-scale multi-path fading are considered in the channel mode. Without loss of generation, we assume that all channels are quasi-static flat block fading. That is, the channels keep constant over one time slot, but may vary from one time slot to the 
next. Please note that the links from MPS to $S$ (denoted as MPS-to-S), and from $S$ to $\mathrm{U}_{k}$ (denoted as S-to- $U_{k}$ ) are assumed to be independent and identically distributed (i.i.d.) Nakagami- $m$ fading.

The channels from MPS to $\mathrm{S}$ and from $\mathrm{S}$ to $\mathrm{U}_{k}$ are denoted by $\boldsymbol{h} \in \mathcal{C}^{N_{\mathrm{t}} \times 1}$ and $\boldsymbol{g}_{k} \in \mathcal{C}^{N_{k} \times 1}$, respectively, where $\boldsymbol{h}=\left[h_{1}, h_{2}, \ldots, h_{N_{\mathrm{t}}}\right]^{\mathrm{T}}$ and $\boldsymbol{g}_{k}=\left[g_{k, 1}, g_{k, 2}, \ldots, g_{k, N_{k}}\right]^{\mathrm{T}}$. The channel gain $\left|h_{i}\right|^{2}$ of the MPS-to-S links follows Gamma distribution with mean $\mu_{\mathrm{t}}$, shape parameter $m_{t}$ and rate parameter $\lambda_{t}=\frac{m_{\mathrm{t}}}{\mu_{\mathrm{t}}}$, i.e., $\left|h_{i}\right|^{2} \sim \operatorname{Gamma}\left(m_{\mathrm{t}}, \lambda_{\mathrm{t}}\right), \forall i=1, \ldots, N_{\mathrm{t}}$. Similarity, the channel gain $\left|g_{k, j}\right|^{2}$ of the S-to- $\mathrm{U}_{k}$ links follows Gamma distribution with parameters $\mu_{k}, m_{k}$ and $\lambda_{k}=\frac{m_{k}}{\mu_{k}}$, i.e., $\left|g_{k, j}\right|^{2} \sim \operatorname{Gamma}\left(m_{k}\right.$, $\left.\lambda_{k}\right), \forall j=1, \ldots, N_{k}$. As a result, it can be derived that $|\boldsymbol{h}|^{2} \sim \operatorname{Gamma}\left(m_{\mathrm{t}} N_{\mathrm{t}}, \lambda_{\mathrm{t}}\right)$ and $\left|\boldsymbol{g}_{k}\right|^{2} \sim$ $\operatorname{Gamma}\left(m_{k} N_{k}, \lambda_{k}\right), \forall k=1, \ldots, K$.

Define $\tilde{h}_{\mathrm{t}}=|\boldsymbol{h}|^{2}$ and $\tilde{h}_{k}=\left|\boldsymbol{g}_{k}\right|^{2}$. According to [42], the probability density function (PDF) and complementary cumulative distribution function (CCDF) of all $\tilde{h}_{z}(z=\mathrm{t}, 1, \ldots, K)$ can be given by

$$
f_{\tilde{h}_{z}}(x)=\frac{\lambda_{z}^{m_{z} N_{z}}}{\Gamma\left(m_{z} N_{z}\right)} x^{m_{z} N_{z}-1} e^{-\lambda_{z} x}
$$

and

$$
F_{\tilde{h}_{z}}(x)=e^{-\lambda_{z} x} \sum_{n=0}^{m_{z} N_{z}-1} \frac{\left(\lambda_{z} x\right)^{n}}{n !}
$$

respectively.

Without loss of generality, it is assumed that the knowledge of MPS-to-S and S-to- $\mathrm{U}_{k}$ channels are available to MPS and $U_{k}$. To enhance the energy efficiency of WPT from MPS to S, MPS uses multiple antennas to transmit the energy RF signal to $S$ via beamforming technology. Specifically, maximum ratio transmission (MRT) is adopted at MPS with a beamforming vector $\boldsymbol{w}_{\mathrm{S}}=\frac{\boldsymbol{h}}{\|\boldsymbol{h}\|^{2}}$, which is used for maximizing the amount of energy harvested at $\mathrm{S}$ [43]. For the multi-antenna users, i.e., $\mathrm{U}_{k}$, maximum ratio combining (MRC) is employed at $\mathrm{U}_{k}$ with a combining weight vector $\boldsymbol{w}_{\mathrm{U}_{k}}=\boldsymbol{g}$, which is used to maximize the instantaneous signal-to-noise ratio (SNR) of the combined signal [42].

\subsection{Linear and Nonlinear EH Models}

For the linear EH model, the harvested energy can be calculated by

$$
\mathrm{Q}_{\mathrm{L}}\left(P_{\text {in }}\right)=\eta P_{\text {in }}
$$

where $\eta \in[0,1]$ is the energy conversion efficiency, and the harvested energy $\mathrm{Q}_{\mathrm{L}}$ linearly and indefinitely increases as the input power $P_{\text {in }}$.

However, the energy harvested by the practical EH circuits are not simply in linearity but in nonlinearity with the input power $P_{\mathrm{in}}$. To accurately model the nonlinearity of practical EH circuits, the LG nonlinear EH model was presented in [23]. That is

$$
\mathrm{Q}_{\mathrm{nL}}\left(P_{\mathrm{in}}\right)=\frac{\frac{M}{1+\exp \left(-a\left(P_{\mathrm{in}}-b\right)\right)}-\frac{M}{1+\exp (a b)}}{1-\frac{1}{1+\exp (a b)}}=\frac{M\left(1-e^{-a P_{\mathrm{in}}}\right)}{1+e^{-a\left(P_{\mathrm{in}}-b\right)}} .
$$

where $M, a$ and $b$ are constants. $M$ is the maximum harvested energy at the receiver when $\mathrm{EH}$ circuit is saturated. $a$ and $b$ are constants. Specifically, $a$ reflects the nonlinear EH rate w.r.t the input power, i.e., $P_{\text {in }} . b$ is related to the minimum turn-on voltage of the EH circuit. That is, $a$ and $b$ correspond to the resistance, the capacitance and the circuit sensitivity, so that they are associated with the specification of EH circuits.

To show the difference between the two models, in Figure 2, we compare the amount of energy harvested at the receiver with them. It can be seen that with the LG nonlinear EH model, when the input power is relatively large, the energy harvested at receiver keep constant because the $\mathrm{EH}$ circuit enters the 
saturation region. Moreover, when the input power is relatively small, the results under the linear EH model with an appropriate $\eta$ may be close to that under the LG nonlinear EH model.

Therefore, to characterize the amount of the energy harvested at MPS and $\mathrm{U}_{k}$ more practically, the LG nonlinear EH model is adopted at MPS in this paper due to relatively large received RF power with WPT [16], and the linear and LG nonlinear EH models are considered at $\mathrm{U}_{k}$ because the amount of the harvested energy is generally small with SWIPT $[2,7]$.

\subsection{Transmission Protocol}

Let $T$ be the total time period of the system transmission. A WPT-SWIPT protocol is presented, where is $T$ divided into two phases with a time assignment factor $0<\alpha<1$. The first phase is the WPT phase with time interval $\alpha T$, and the second one is the SWIPT phase with time interval $(1-\alpha) T$, as shown in Figure 3.

\begin{tabular}{|c|c|}
\hline Phase 1: $\alpha \mathrm{T}$ & Phase 2: $(1-\alpha) \mathrm{T}$ \\
\hline $\begin{array}{l}\text { MPS transmits power to } \mathrm{S}, \mathrm{S} \\
\text { is for } \mathrm{EH} \text {. }\end{array}$ & $\begin{array}{c}S \text { transmits information and } \\
\text { power to } U_{k} \text { simultaneously, } \\
\mathrm{U}_{k} \text { is for ID and EH. }\end{array}$ \\
\hline
\end{tabular}

Figure 3. Transmission protocol: WPT-SWPT protocol.

\subsubsection{EH at $\mathrm{S}$}

In the WPT phase with time interval $\alpha T$, MPS transmits power to $S$, and then $S$ harvests energy from the received RF signals. The nonlinear $\mathrm{EH}$ model is adopted at $\mathrm{S}$, so the energy harvested at $\mathrm{S}$ from MPS can be given by

$$
\mathrm{Q}_{\mathrm{s}}=\alpha T \mathrm{Q}_{\mathrm{nL}}\left(P_{\mathrm{t}} \tilde{h}_{\mathrm{t}}\right)
$$

where $P_{\mathrm{t}}$ is the transmit power of MPS.

\subsubsection{ID at $\mathrm{U}_{k}$}

In the SWIPT phase with time interval $(1-\alpha) T, \mathrm{~S}$ consumes all harvested energy to broadcast information and power RF signals to $K$ uses at the same time. Please note that we assume that a power amplifier with efficiency $0<\zeta<1$ is employed at $S$ to deal with its transmit power. The transmit power at $\mathrm{S}$ is given by

$$
P_{\mathrm{s}}=\frac{\zeta \mathrm{Q}_{\mathrm{s}}}{(1-\alpha) T}=\frac{\zeta \alpha \mathrm{Q}_{\mathrm{nL}}\left(P_{\mathrm{t}} \tilde{h}_{\mathrm{t}}\right)}{(1-\alpha)}
$$

Then, the PS receiver architecture is adopted at $\mathrm{U}_{k}$ with a PS ratio $0 \leq \rho_{k} \leq 1$ as shown in Figure 1 , where the $\rho_{k}$ part of the received RF power is used for ID and the rest part is used for EH. Thus, the received $R F$ signals at $\mathrm{U}_{k}$ can be given by

$$
y_{k}=\sqrt{\rho_{k} P_{\mathrm{s}}} g_{k} x+n_{k}
$$

where $n_{k}$ is additive white gaussian noise generated by ID with mean zero and variance $\sigma_{k}^{2}$ at $\mathrm{U}_{k}$. Therefore, the received $\mathrm{SNR}$ at $\mathrm{U}_{k}$ is given by

$$
\gamma_{k}=\frac{\rho_{k} P_{\mathrm{s}}\left|g_{k}\right|^{2}}{\sigma_{k}^{2}}=\frac{\rho_{k} \zeta \alpha \mathrm{Q}_{\mathrm{nL}}\left(P_{\mathrm{t}} \tilde{h}_{\mathrm{t}}\right) \tilde{h}_{k}}{(1-\alpha) \sigma_{k}^{2}} .
$$




\subsection{3. $\mathrm{EH}$ at $\mathrm{U}_{k}$}

The $\left(1-\rho_{k}\right)$ part of power is used for $E H$, so the received RF power at $\mathrm{U}_{k}$ for $\mathrm{EH}$ from $\mathrm{S}$ can be given by

$$
E_{k}=\rho_{k} P_{\mathrm{s}}\left|g_{k}\right|^{2}=\frac{\left(1-\rho_{k}\right) \zeta \alpha \mathrm{Q}_{\mathrm{nL}}\left(P_{\mathrm{t}} \tilde{h}_{\mathrm{t}}\right) \tilde{h}_{k}}{(1-\alpha)}
$$

If the linear $\mathrm{EH}$ model in (3) at $\mathrm{U}_{k}$ is adopted, the total energy harvested at $\mathrm{U}_{k}$ from $\mathrm{S}$ is given by

$$
Q_{\mathrm{L}, k}=\eta(1-\alpha) T E_{k} .
$$

If the nonlinear $\mathrm{EH}$ model in (4) at $\mathrm{U}_{k}$ is adopted, the total energy harvested at $\mathrm{U}_{k}$ from $\mathrm{S}$ is given by

$$
Q_{\mathrm{nL}, k}=(1-\alpha) T \mathrm{Q}_{\mathrm{nL}}\left(E_{k}\right) .
$$

\section{Outage Probability and Throughput Analysis}

In this section, we analyze the outage performance of the system and a closed-form expression of the system outage probability is obtained.

\subsection{General Outage Probability and Throughput Analysis}

Let the transmission rate requirement at each user be $R_{0}$, the corresponding SNR threshold $\gamma_{0}$ can be given by $\gamma_{0}=2^{R_{0}}-1$. The system outage event occurs when SNR $\gamma_{k}$ at $U_{k}$ is less than SNR threshold $\gamma_{0}$, i.e., $\gamma_{k}<\gamma_{0}, \forall k=1, \ldots, K$.

Proposition 1. The average outage probability $P_{\mathrm{o}, k}$ and the reliable throughput $\mathcal{T}$ of the system are given by

$$
\mathcal{P}_{\mathrm{o}}=1-\prod_{k=1}^{K} \int_{0}^{\infty} F_{\tilde{h}_{k}}(\Delta(x)) f_{\tilde{h}_{\mathrm{t}}}(x) d x,
$$

and

$$
\mathcal{T}=(1-\alpha) T R_{0} \prod_{k=1}^{K} \int_{0}^{\infty} F_{\tilde{h}_{k}}(\Delta(x)) f_{\tilde{h}_{\mathrm{t}}}(x) d x
$$

respectively, where $\Delta(x)=\frac{\delta\left(1+e^{a b}\right)}{1-e^{-a P_{\mathrm{t}} x}}-\delta e^{a b}$ with $\delta=\frac{\gamma_{0}(1-\alpha) \sigma_{k}^{2}}{\rho_{k} \zeta M \alpha}$.

Proof. See AppendixA.

\subsection{Asymptotic Outage Probability and Throughput Analysis}

To reduce the computational complexity, the asymptotic expressions of the outage probability and reliable throughput for the system are discussed for high-transmit power scenarios.

Proposition 2. The asymptotic outage probability and reliable throughput for the system are given by

$$
\lim _{P_{\mathrm{t}} \rightarrow \infty} \mathcal{P}_{\mathrm{o}}^{(\text {asy })}=1-\prod_{k=1}^{K} \frac{\Gamma\left(m_{k} N_{k}, \lambda_{k} \delta\right)}{\Gamma\left(m_{k} N_{k}\right)}
$$

and

$$
\lim _{P_{\mathrm{t}} \rightarrow \infty} \mathcal{T}^{\text {(asy) }}=(1-\alpha) T R_{0} \prod_{k=1}^{K} \frac{\Gamma\left(m_{k} N_{k}, \lambda_{k} \delta\right)}{\Gamma\left(m_{k} N_{k}\right)}
$$


respectively, where $\Gamma(\cdot, \cdot)$ is the upper incomplete gamma function.

Proof. See Appendix B.

\section{Simulation and Results}

In this section, some numerical and simulation results are presented to discuss the outage and reliable throughput performance of the system under the LG nonlinear and linear EH models. Unless specifically stated, the number of users is set as $K=2$, the PS ratio for each user is $\rho_{k}=0.1$, and the noise at user is assumed as $\sigma_{k}^{2}=-50 \mathrm{dBm}$. The time assignment factor for the system is set to be $\alpha=0.7$. For the Nakagami- $m$ channel model, $m$ is set to be 2 for all channels. The power amplifier factor is $\zeta=0.75$. For the LG nonlinear EH model, we set $M=24 \mathrm{~mW}, a=150$ and $b=0.014$ as presented in [30]. For comparison, we set $\eta=0.8$ for the linear EH model. Without loss of generality, the system throughput requirement is set to be $R_{0}=5 \mathrm{bits} / \mathrm{s} / \mathrm{Hz}$.

Figure 4 shows the effect of the transmit power $P_{\mathrm{t}}$ at MPS on the system outage probability with different antenna numbers at MPS and $\mathrm{U}_{k}$, i.e., $\left\{N_{\mathrm{t}}, N_{k}\right\}=\{\{1,2,3\},\{1,2,3\}\}$. It is seen that the results of theoretical analysis match the simulation results obtained by Mont Carlo measurement with $10^{5}$ realizations very well, which verify the correctness of our theoretical analysis. Moreover, as the numbers of antennas increase at MPS and $\mathrm{U}_{k}$, the decreasing rate of the system outage probability increases due to the gain of multiple antennas. At the same time, the system outage probability decreases with the increment of $P_{\mathrm{t}}$, because large $P_{\mathrm{t}}$ usually leads to high SNR. Moreover, when $P_{\mathrm{t}}$ is large enough, i.e., $P_{t} \rightarrow \infty$, the system outage probability is not affected by the number of antennas at MPS but is affected by the number of antennas at $U_{k}$, which is consistent with the asymptotic outage probability of the system in (14).

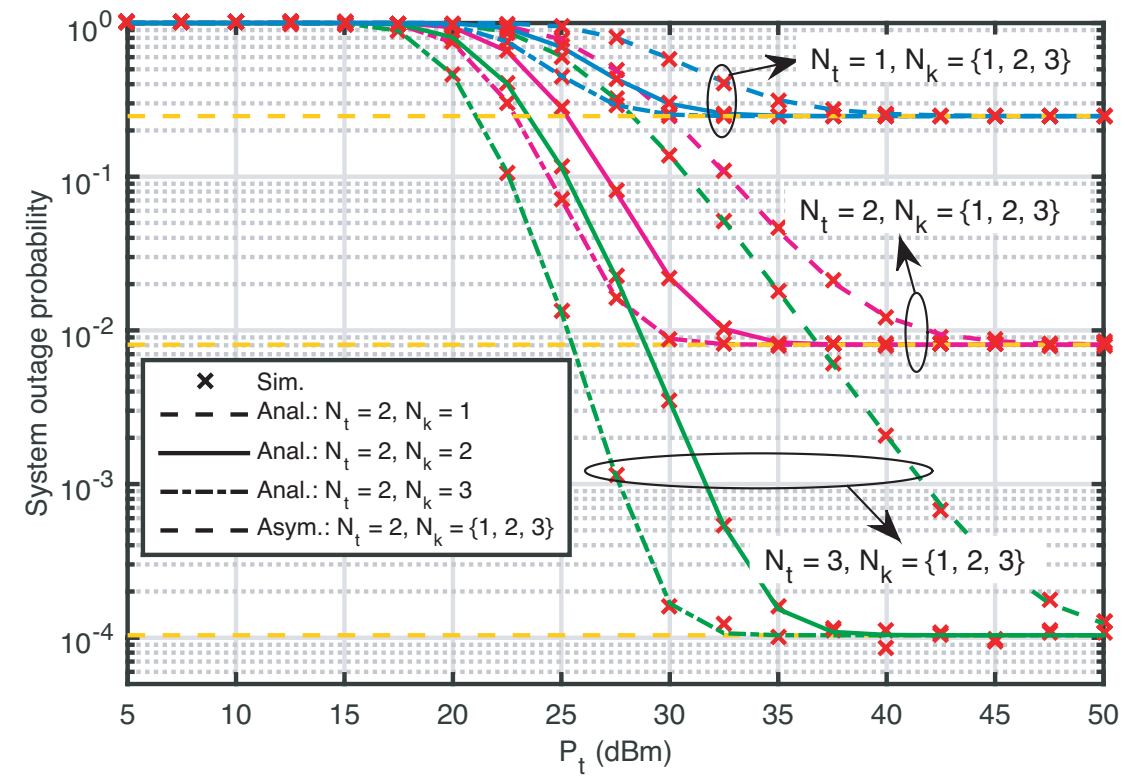

Figure 4 . The system outage probability vs. $P_{\mathrm{t}}$ at MPS.

Figure 5 plots the system reliable throughput versus the transmit power $P_{\mathrm{t}}$ at MPS, where $\left\{N_{\mathrm{t}}, N_{k}\right\}=\{3,\{1,2,3\}\}$. The results of the system reliable throughput respond to the results of the system outage probability associated with $\left\{N_{\mathrm{t}}, N_{k}\right\}=\{3,\{1,2,3\}\}$ in Figure 4 . It can be seen that the system reliable throughput is improved as the antenna numbers $N_{k}$ at $\{\mathrm{U}\}_{\mathrm{k}}$ increases, because the system outage probability is reduced because of the multiple antenna gain at $\mathrm{U}_{k}$. 


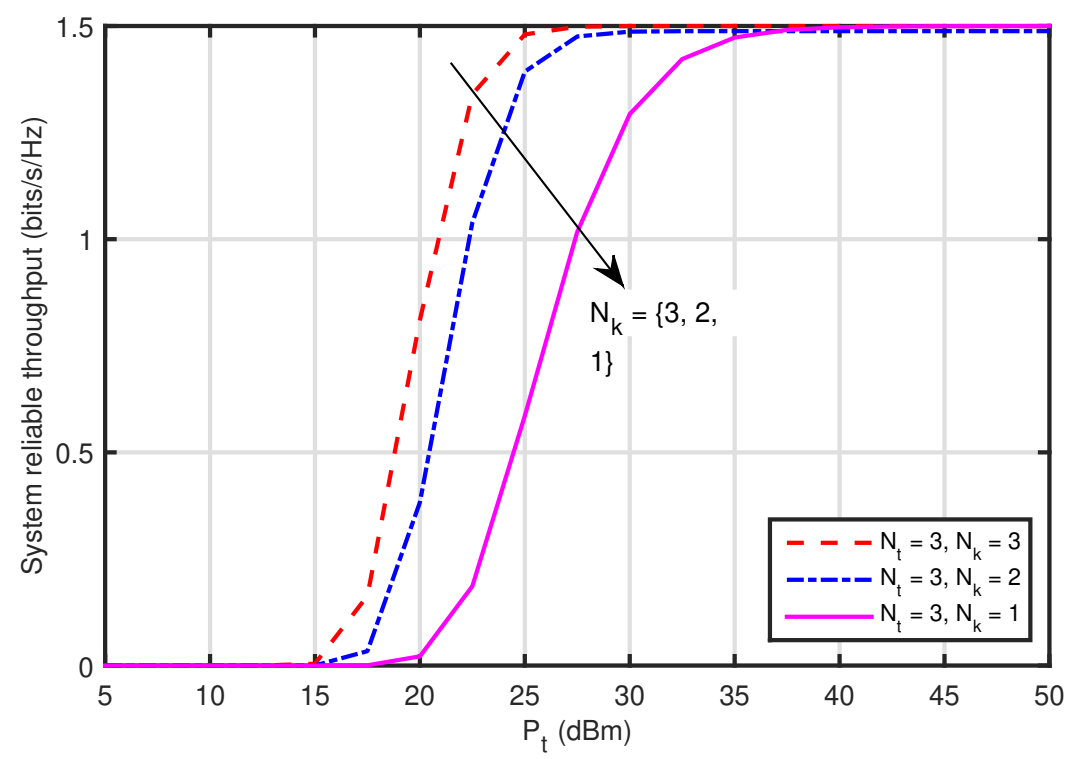

Figure 5. The system reliable throughput vs. $P_{\mathrm{t}}$.

Figure 6 plots the system reliable throughput versus the transmission time factor $\alpha$ with different antenna numbers at MPS and $U_{k}$ in high SNR region. It is observed that the system reliable throughput first increases and then decreases as the increment of $\alpha$, because when $\alpha$ is large, the time for the WPT phase is long but for the SWIPT phase is short. Particularly, the information transmission in SWIPT phase is reduced, so that the throughput of the system decreases, and vice versa. Moreover, the system reliable throughput is improved by increasing the number of antennas at $\mathrm{U}_{k}$ but at MPS. That is why the reliable throughput in (14) is just related to $N_{k}$, and more antenna leads to a lower system outage probability. Moreover, when the antenna numbers at $U_{k}$ are different, i.e., $N_{k}=\{1,2,3\}$, the achieved optimal transmission time factors are different, i.e., $\alpha=\{0.67,0.48,0.36\}$. When $\alpha$ is larger than the optimal one, the system reliable throughput trends to decrease and then constant. That is why when $\alpha$ is larger, the time for information transmission is less in the SWIPT phase.

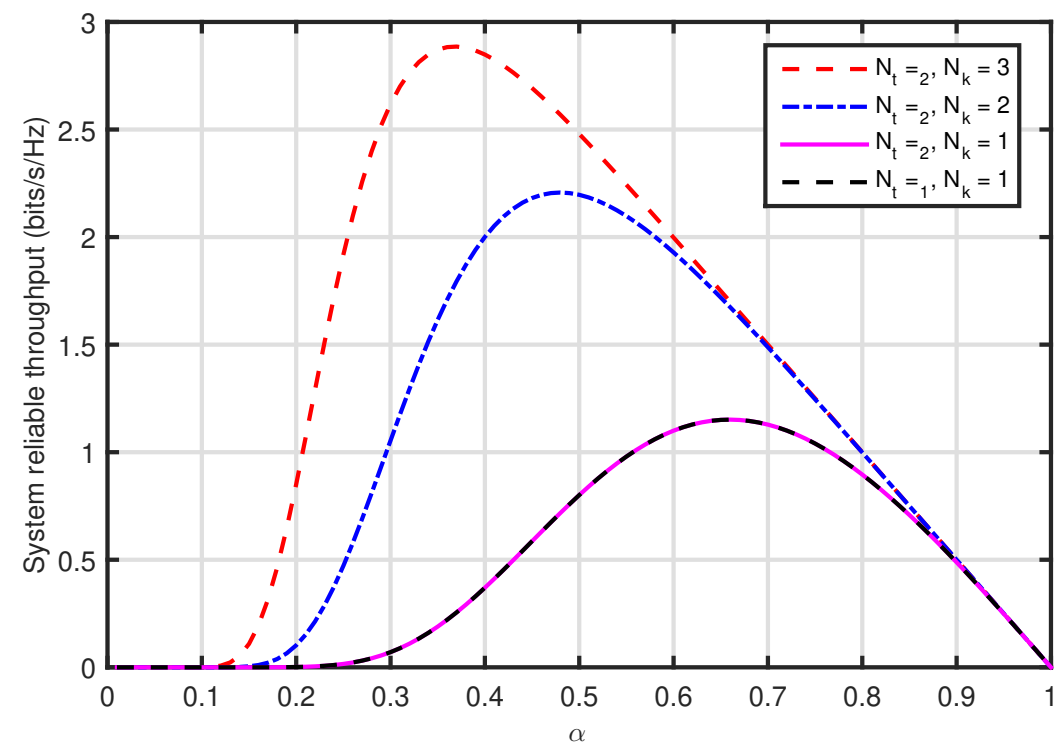

Figure 6. The system throughput vs. the transmission time factor $\alpha$, where $P_{\mathrm{t}} \rightarrow \infty$. 
Figure 7 shows the system reliable throughput versus the transmission rate pre-threshold $R_{0}$ at each user with different antenna number at MPS and $\mathrm{U}_{k}$. It is seen that the system reliable throughput first increases and then decreases as $R_{0}$ increases. When $R_{0}$ is less than $3.2 \mathrm{bits} / \mathrm{s} / \mathrm{Hz}$, the average throughputs of the system with different antenna numbers are almost identical, because the system outage probability approaches zero when $R_{0}$ is relatively low. Moreover, the different optimal $R_{0}$ can be achieved for different systems associated with $N_{k}=\{1,2,3\}$, the corresponding optimal $R_{0}$ is $\{4.7,5.9,6.8\}$ bits/s/Hz.

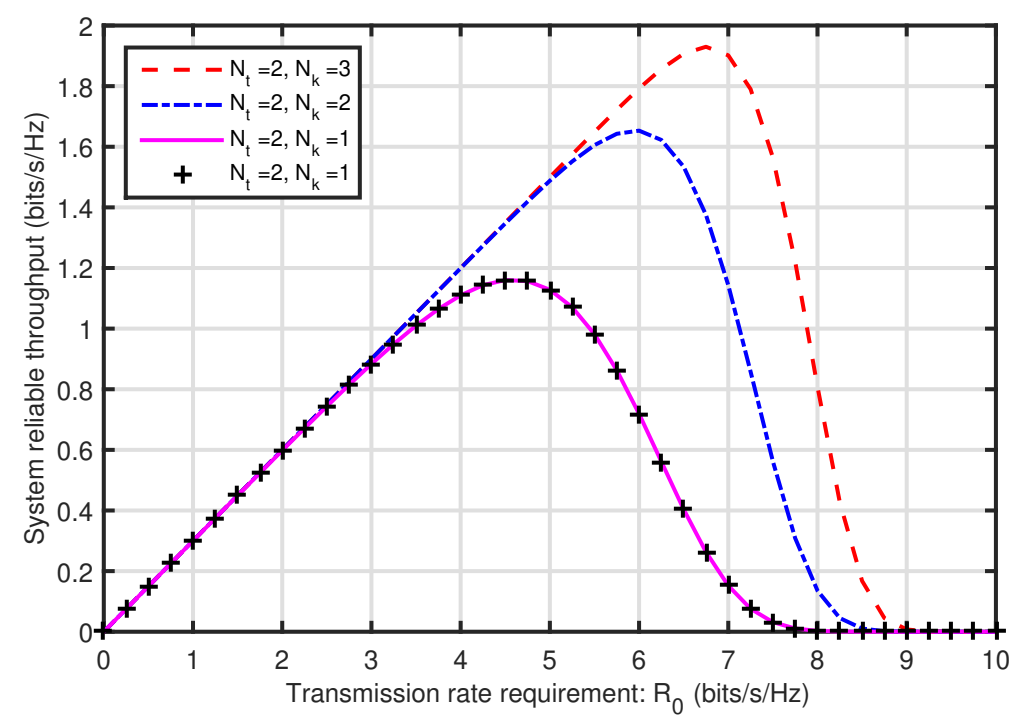

Figure 7. The reliable throughput vs. the transmission rate requirement $R_{0}$ at each user, where $P_{\mathrm{t}} \rightarrow \infty$.

Figure 8 compares the impact of the shape parameter of Nakagami- $m$ channels $m$ on the system outage probability versus the transmit power $P_{\mathrm{t}}$ at MPS. It shows that the system outage probability associated with $m_{1}=1$ and $m_{k}=1$ is largest, because when $m_{1}=1$ and $m_{k}=1$, the transmission channels follow the Rayleigh fading. Moreover, the system outage probability is decreased with the increment of $m_{k}$ when $m_{\mathrm{t}}=1$, and vice versa. That is, $m$ is larger, the system outage probability is lower.

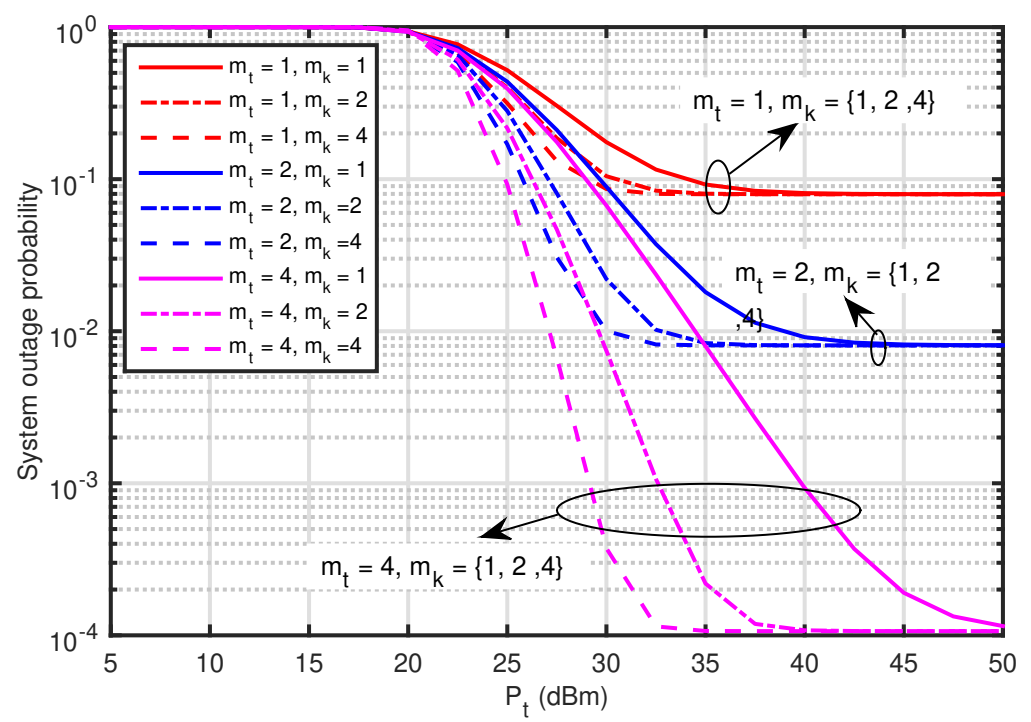

Figure 8. The system outage probability vs. the shape parameter of Nakagami- $m$ channels $m$. 
Figure 9 shows the region between the system reliable throughput and the amount of energy harvested at $\mathrm{U}_{k}$ with different transmit power $P_{\mathrm{t}}$ at MPS, i.e., $\mathcal{T}$ - $\mathrm{Q}_{k}$ region. To compare the impact of adopting the linear and LG nonlinear EH models at $\mathrm{U}_{k}$ on the $\mathcal{T}-\mathrm{Q}_{k}$ region, we set $\eta=\{0.4,0.5\}$ for the linear EH model. It can be observed that the $\mathcal{T}-\mathrm{Q}_{k}$ region under the $\mathrm{LG}$ nonlinear EH model is smaller than that under the linear one with $\eta=0.5$. While, when $\eta=0.4$ and $P_{\mathrm{t}}=20 \mathrm{dBm}$, the region is the same for the two EH models. That is, when $\eta=0.4$, the same system performance can be obtained under both EH models. That is why the achievable RF power at $\mathrm{U}_{k}$ is relatively low, the results under the linear EH model is closed to that under the LG nonlinear one, which is also observed in Figure 2. When $P_{\mathrm{t}}=25$ and $45 \mathrm{dBm}$, the regions with both $\mathrm{EH}$ models are different due to the saturation characteristics of the EH circuit. Since the LG nonlinear EH model is closer to the features of practical EH circuit than the linear one, using the linear EH model causes the false achievable system performance output. This observation is consistent to that in [23-34].

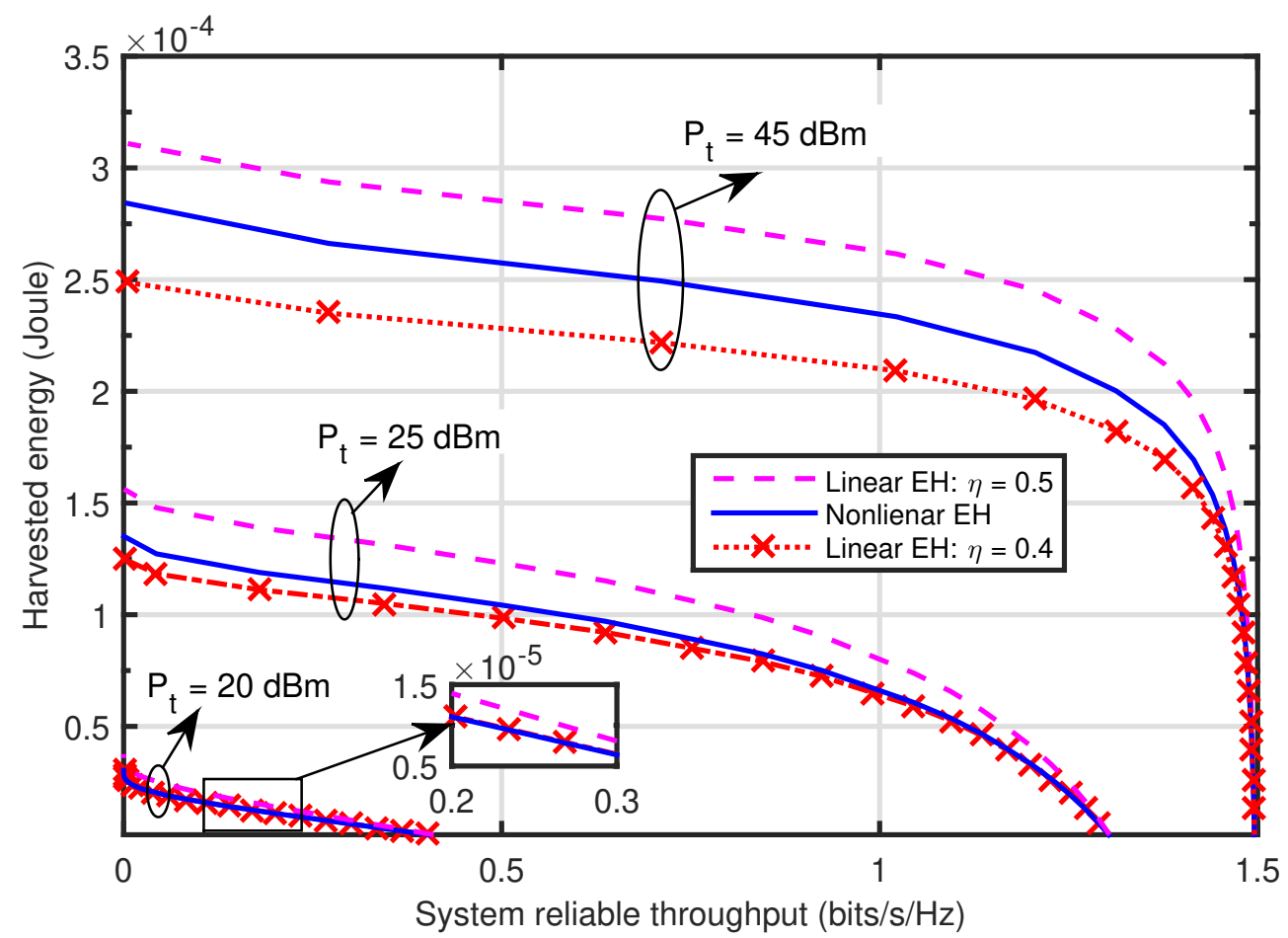

Figure 9. The region between the reliable throughput and the amount of energy harvested at each MUs, where $N_{0}=2, N_{k}=3$, and $K=1$.

\section{Conclusions}

This paper studied the outage probability and reliable throughput of a multi-user WPCN-SWIPT system in Nakagami- $m$ fading. For the system, the LG nonlinear EH model was adopted to analyze the system performance and for comparison, the system performance with the linear EH model was also analyzed. The closed-form expressions of the system outage probability were derived. Moreover, in the high-transmit power scenarios, the asymptotic expressions of the system outage probability were also presented. Moreover, the expressions of the system reliable throughput were also achieved. Simulation results demonstrate the correctness of our derived analytical results and show that the systems under the LG nonlinear and linear EH models have very different performance behaviors. Using the LG nonlinear EH model can avoid false results of system performance since the LG nonlinear EH model is closer to the features of practical EH circuit than the linear one. 
Author Contributions: Conceptualization, Methodology, Investigation, Writing-Original Draft Preparation, Simulation, K.X. and R.J. (equally contributed); Writing-Review \& Editing, Y.Z. and L.Z.; Funding Acquisition, Y.Z.; Resources, T.L. Supervision, K.X. and Z.Z.

Funding: This work was supported in part by the National Key R\&D Program of China (No. 2016YFE0200900), in part by the General Program of the National Natural Science Foundation of China (NSFC) (No. 61671051), in part by the Beijing Intelligent Logistics System Collaborative Innovation Center (No. BILSCIC-2018KF-05), in part by the Young Talents Program of State Grid Energy Research Institute Co., Ltd. (Research on the key technology of fog computing for smart grid, No. XM2018020035180) and also in part by the Fundamental Research Funds for the Central Universities (No. 2017YJS062).

Conflicts of Interest: The authors declare no conflict of interest.

\section{Abbreviations}

The following abbreviations are used in this manuscript:

WPCN Wireless-powered communication network

SWIPT Simultaneous wireless information and power transfer

LG Logistic

EH Energy-harvesting

PS Power-splitting

WDs Wireless devices

WSNs Wireless sensor networks

IoT Internet of Things

WPAN Wireless personal area networks

RF Radio-frequency

WIT Wireless information transfer

WPT Wireless power transfer

ID Information decoding

TS Time switching

AF Amplify-and-forward

DF Decode-and-forward

PW Piecewise

FR Fractional

S Source

MPS Multi-antenna power station

$\mathrm{U}_{k} \quad$ the $k$-th user

FS fog server

PDF Probability density function

CCDF Complementary cumulative distribution function

MRT Maximum rate transmission

MRC Maximum ratio combining

SNR Signal-to-noise ratio

\section{Appendix A. Proof of Proposition 1}

Proof. With (8), the average outage probability $\mathcal{P}_{\mathrm{o}, k}$ at $\mathrm{U}_{k}$ by averaging over $\tilde{h}_{t}$ is expressed as

$$
\mathcal{P}_{\mathrm{o}, k}=\operatorname{Pr}\left(\gamma_{k}<\gamma_{0}\right)=\operatorname{Pr}\left(\frac{\rho_{k} \zeta \alpha \mathrm{Q}_{\mathrm{nL}}\left(P_{\mathrm{t}} \tilde{h}_{\mathrm{t}}\right) \tilde{h}_{k}}{(1-\alpha) \sigma_{k}^{2}}<\gamma_{0}\right)=\operatorname{Pr}\left(\tilde{h}_{k}<\Delta\left(\tilde{h}_{\mathrm{t}}\right)\right)=1-F_{\tilde{h}_{k}}\left(\Delta\left(\tilde{h}_{\mathrm{t}}\right)\right)
$$


where $\Delta\left(\tilde{h}_{\mathrm{t}}\right)=\frac{\delta\left(1+e^{a b}\right)}{1-e^{-\mathrm{aP}_{\mathrm{t}} \hat{h}_{\mathrm{t}}}}-\delta e^{a b}$ with $\delta=\frac{\gamma_{0}(1-\alpha) \sigma_{k}^{2}}{\rho_{k} \zeta M \alpha}$. With the CCDF and PDF of $\tilde{h}_{t}$ in (2) and the fact $\int_{0}^{\infty} f_{\tilde{h}_{\mathrm{t}}}(x) d x=1, \mathcal{P}_{\mathrm{o}, k}$ in (A1) can be further given by

$$
\begin{aligned}
\mathcal{P}_{\mathrm{o}, k} & =\int_{0}^{\infty}\left(1-F_{\tilde{h}_{k}}(\Delta(x))\right) f_{\tilde{h}_{\mathrm{t}}}(x) d x=\int_{0}^{\infty} f_{\tilde{h}_{\mathrm{t}}}(x) d x-\int_{0}^{\infty} F_{\tilde{h}_{k}}(\Delta(x)) f_{\tilde{h}_{\mathrm{t}}}(x) d x \\
& =1-\int_{0}^{\infty} F_{\tilde{h}_{k}}(\Delta(x)) f_{\tilde{h}_{\mathrm{t}}}(x) d x .
\end{aligned}
$$

Since each user is independent and does not interfere with each other, the outage probability for every user is the same. Therefore, the system outage probability with multiple users is given by

$$
\mathcal{P}_{\mathrm{o}}=1-\left(1-P_{\mathrm{o}, 1}\right)\left(1-P_{\mathrm{o}, 2}\right) \cdots\left(1-\mathcal{P}_{\mathrm{o}, k}\right)
$$

Then, based on the outage probability of $\mathrm{U}_{k}$ in (A2), the outage probability of the system in (A3) can be further expressed as

$$
\mathcal{P}_{\mathrm{o}}=1-\prod_{k=1}^{K} \int_{0}^{\infty} F_{\tilde{h}_{k}}(\Delta(x)) f_{\tilde{h}_{\mathrm{t}}}(x) d x .
$$

Thus, the system outage probability in (12) is obtained. If the system does not interrupt, the system throughput is expressed as

$$
\mathcal{T}=(1-\alpha) T R_{0}
$$

While, in the practical communication environment, the system has a certain outage probability. The throughput of the system is reliable only when the probability of system interruption is considered. Therefore, based on the analysis of the system outage probability mentioned above, the system reliable throughput can be given by

$$
\mathcal{T}=(1-\alpha) T\left(1-\mathcal{P}_{\mathrm{o}}\right) R_{0}
$$

Substituting (A3) into (A6), the system reliable throughput in (13) can be achieved. Thus, the proof ends.

\section{Appendix B. Proof of Proposition 2}

Proof. With the LG nonlinear EH model, when $P_{\mathrm{t}} \rightarrow \infty$, the maximum harvested energy is $\mathrm{Q}_{\mathrm{nL}}\left(P_{\mathrm{in}}\right) \rightarrow M$. Based on this and (8), the outage probability $\mathcal{P}_{\mathrm{o}, k}$ at $\mathrm{U}_{k}$ can be given by

$$
\mathcal{P}_{\mathrm{o}, k}=\operatorname{Pr}\left(\gamma_{k}<\gamma_{0}\right)=\operatorname{Pr}\left(\frac{\rho_{k} \zeta \alpha M \tilde{h}_{k}}{(1-\alpha) \sigma_{k}^{2}}<\gamma_{0}\right)=\operatorname{Pr}\left(\tilde{h}_{k}<\frac{\gamma_{0}(1-\alpha) \sigma_{k}^{2}}{\rho_{k} \zeta \alpha M}\right)=\operatorname{Pr}\left(\tilde{h}_{k}<\delta\right)
$$

Then, by using the CCDF of $\tilde{h}_{k}$ in (2), the asymptotic outage probability at $\mathrm{U}_{k}$ is expressed as

$$
\lim _{P_{\mathrm{t}} \rightarrow \infty} \mathcal{P}_{\mathrm{o}, k}^{(\text {asy })}=\operatorname{Pr}\left(\frac{\rho_{k} \alpha \zeta M \tilde{h}_{k}}{(1-\alpha) \sigma_{k}^{2}}<\gamma_{0}\right)=1-F_{\tilde{h}_{k}}(\delta) .
$$

Following (A3) and (A4), the asymptotic outage probability of the system can be given by

$$
\lim _{P_{\mathrm{t}} \rightarrow \infty} \mathcal{P}_{\mathrm{o}}^{(\text {asy })}=1-\prod_{k=1}^{K}\left(1-\left(1-F_{\tilde{h}_{k}}(\delta)\right)\right)=1-\prod_{k=1}^{K} e^{-\lambda_{k} \delta} \sum_{n=0}^{m_{k} N_{k}-1} \frac{\left(\lambda_{k} \delta\right)^{n}}{n !} .
$$


With the fact $\frac{\Gamma(s, x)}{\Gamma(s)}=\frac{(s-1) ! e^{-x} \sum_{k=0}^{s-1} \frac{x^{k}}{k !}}{(s-1) !}=e^{-x} \sum_{k=0}^{s-1} \frac{x^{k}}{k !}[44-46]$, the system asymptotic outage probability in (A9) is further given by

$$
\lim _{P_{\mathrm{t}} \rightarrow \infty} \mathcal{P}_{\mathrm{o}}^{\text {(asy) }}=1-\prod_{k=1}^{K} e^{-\lambda_{k} \delta} \sum_{n=0}^{m_{k} N_{k}-1} \frac{\left(\lambda_{k} \delta\right)^{n}}{n !}=1-\prod_{k=1}^{K} \frac{\Gamma\left(m_{k} N_{k}, \lambda_{k} \delta\right)}{\Gamma\left(m_{k} N_{k}\right)} .
$$

where $\Gamma(\cdot, \cdot)$ is the upper incomplete gamma function. Therefore, the asymptotic outage probability in (14) is derived. Similar to the proof of the system reliable throughput in (13), and following (A6), the asymptotic reliable throughput of the system can be expressed as

$$
\lim _{P_{\mathrm{t}} \rightarrow \infty} \mathcal{T}^{\text {(asy) }}=(1-\alpha) T\left(1-\mathcal{P}_{\mathrm{o}}^{(\text {asy })}\right) R_{0} .
$$

Substituting $\mathcal{P}_{\mathrm{o}}^{(\text {asy) }}$ in (A10) into (A11), the asymptotic reliable throughput of the system in (15) can be achieved. Thus, the proof ends.

\section{References}

1. Bassoli, M.; Bianchi, V.; Munari, I.D. A plug and play IoT wi-fi smart home system for human monitoring. Electronics 2018, 7, 200. [CrossRef]

2. Urmonov, O.; Kim, H. An energy-efficient fail recovery routing in TDMA MAC protocol-based wireless sensor network. Electronics 2018, 7, 444. [CrossRef]

3. Cao, J.; Bu, Z.; Gao, G.; Tao, H. Weighted modularity optimization for crisp and fuzzy community detection in large-scale networks. Phys. A Stat. Mech. Appl. 2016, 462, 386-395. [CrossRef]

4. Liu, J.; Zha, L.; Cao, J.; Fei, S. Hybrid-driven-based stabilisation for networked control systems. IET Control Theory Appl. 2016, 10, 2279-2285. [CrossRef]

5. Wang, L.; Hu, F.; Ling, Z.; Wang, B. Wireless information and power transfer to maximize information throughput in WBAN. IEEE Internet Things J. 2017, 4, 1663-1670. [CrossRef]

6. Valenta, C.R.; Durgin, G.D. Harvesting wireless power: Survey of energy-harvester conversion efficiency in far-field, wireless power transfer systems. IEEE Microw. Mag. 2014, 15, 108-120.

7. Xiong, K.; Fan, P.; Zhang, C.; Letaief, K.B. Wireless information and energy transfer for two-hop non-regenerative MIMO-OFDM relay networks. IEEE J. Sel. Areas Commun. 2015, 33, 1595-1611. [CrossRef]

8. Xiong, K.; Chen, C.; Qu, G.; Fan, P.; Letaief, K.B. Group cooperation with optimal resource allocation in wireless powered communication networks. IEEE Trans. Wirel. Commun. 2017, 16, 3840-3853. [CrossRef]

9. Lu, X.; Wang, P.; Niyato, D.; Kim, D.I.; Han, Z. Wireless networks with RF energy harvesting: A contemporary survey. IEEE Commun. Surveys Tuts. 2015, 17, 757-789. [CrossRef]

10. Di, X.; Xiong, K.; Fan, P.; Yang, H. Simultaneous wireless information and power transfer in cooperative relay networks with rateless codes. IEEE Trans. Veh. Technol. 2017, 66, 2981-2996. [CrossRef]

11. Ahmed, F.; Ahmed, T.; Muhammad, Y.; Moullec, Y.L.; Annus, P. Operating wireless sensor nodes without energy storage: Experimental results with transient computing. Electronics 2016, 5, 89. [CrossRef]

12. Zeng, Y.; Clerckx, B.; Zhang, R. Communications and signals design for wireless power transmission. IEEE Trans. Wireless Commun. 2017, 65, 2264-2290. [CrossRef]

13. Almohaimeed, A.M.; Amaya, R.E.; Lima, J.A.; Yagoub, M.C.E. An adaptive power harvester with active load modulation for highly efficient short/long range RF WPT applications. Electronics 2018, 7, 125. [CrossRef]

14. Zheng, H.; Xiong, K.; Fan, P.; Zhou, L.; Zhong, Z.S WIPT-aware fog information processing: Local computing vs. fog offloading. Sensors 2018, 18, 3291. [CrossRef]

15. Lu, Y.; Xiong, K.; Fan, P.; Zhong, Z.; Letaief, K.B. Robust transmit beamforming with artificial redundant signals for secure SWIPT system under non-linear EH model. IEEE Trans. Wirel. Commun. 2018, 17, 2218-2232. [CrossRef]

16. Ju, H.; Zhang, R. Throughput maximization in wireless powered communication networks. IEEE Trans. Wireless Commun. 2014, 13, 418-428. [CrossRef]

17. Zhang, R.; Ho, C.K. MIMO broadcasting for simultaneous wireless information and power transfer. IEEE Trans. Wireless Commun. 2013, 12, 1989-2001. [CrossRef] 
18. Nasir, A.A.; Zhou, X.; Durrani, S.; Kennedy, R.A. Relaying protocols for wireless energy harvesting and information processing. IEEE Trans. Wireless Commun. 2013, 12, 3622-3636. [CrossRef]

19. Jiang, R.; Xiong, K.; Zhang, Y.; Zhong, Z. Outage analysis and optimization of SWIPT in network-coded two-way relay networks. Mob. Inf. Syst. 2017, 2017, 16p. [CrossRef]

20. Mukherjee, A.; Acharya, T.; Khandaker, M.R.A. Outage analysis for SWIPT-enabled two-way cognitive cooperative communications. IEEE Trans. Veh. Technol. 2018, 67, 9032-9036. [CrossRef]

21. Majid Butt, M.; Salama, A.; Mohamed, A.; Guizani, M. Relay selection schemes to minimise outage in wireless powered communication networks. IET Signal Process. 2016, 10, 203-209. [CrossRef]

22. Moon, J.; Lee, H.; Song, C.; Lee, I. Secrecy performance optimization for wireless powered communication networks with an energy harvesting jammer. IEEE Trans. Wirel. Commun. 2017, 65, 764-774. [CrossRef]

23. Boshkovska, E.; Ng, D.W.K.; Zlatanov, N.; Schober, R. Practical non-linear energy harvesting model and resource allocation for SWIPT systems. IEEE Commun. Lett. 2015, 19, 2082-2085. [CrossRef]

24. Boshkovska, E.; Morsi, R.; Ng, D.W.K.; Schober, R. Power allocation and scheduling for SWIPT systems with nonlinear energy harvesting model. In Proceedings of the 2016 IEEE International Conference on Communications (ICC), Kuala Lumpur, Malaysia, 22-27 May 2016; pp. 1-6.

25. Boshkovska, E.; Koelpin, A.; Ng, D.W.K.; Zlatanov, N.; Schober, R. Robust beamforming for SWIPT systems with nonlinear energy harvesting model. In Proceedings of the 2016 IEEE 17th International Workshop on Signal Processing Advances in Wireless Communications (SPAWC), Edinburgh, UK, 3-6 July 2016; pp. 1-5.

26. Kang, J.; Kim, I.; Kim, D.I. Joint optimal mode switching and power adaptation for nonlinear energy harvesting SWIPT system over fading channel. IEEE Trans. Commun. 2018, 66, 1817-1832. [CrossRef]

27. Xiong, K.; Wang, B.; Liu, K.J.R. Rate-energy region of SWIPT for MIMO broadcasting under nonlinear energy harvesting model. IEEE Trans. Wirel. Commun. 2017, 16, 5147-5161. [CrossRef]

28. Jiang, R.; Xiong, K.; Fan, P.; Zhang, Y.; Zhong, Z. Optimal design of SWIPT systems with multiple heterogeneous users under non-linear energy harvesting model. IEEE Access 2017, 5, 11479-11489. [CrossRef]

29. Lu, Y.; Xiong, K.; Fan, P.; Ding, Z.; Zhong, Z.; Letaief, K.B. Global energy efficiency in secure MISO SWIPT systems with non-linear power-splitting EH model. IEEE J. Sel. Areas Commun. 2019, 37, 216-232. [CrossRef]

30. Boshkovska, E.; Ng, D.W.K.; Zlatanov, N.; Koelpin, A.; Schober, R. Robust resource allocation for MIMO wireless powered communication networks based on a non-linear EH model. IEEE Trans. Commun. 2017, 65, 1984-1999. [CrossRef]

31. Venkategowda, N.K.D.; Lee, H.; Lee, I. Joint transceiver designs for MSE minimization in MIMO wireless powered sensor networks. IEEE Trans. Commun. 2018, 17, 5120-5131. [CrossRef]

32. Yang, Z.; Xu, W.; Pan, Y.; Pan, C.; Chen, M. Energy efficient resource allocation in machine-to-machine communications with multiple access and energy harvesting for IoT. IEEE Internet Things J. 2018, 5, 229-245. [CrossRef]

33. Huang, Y.; Li, Z.; Zhou, F.; Zhu, R. Robust AN-aided beamforming design for secure MISO cognitive radio based on a practical nonlinear EH model. IEEE Access 2017, 5, 14011-14019. [CrossRef]

34. Liu, X.; Li, Z.; Wang, C. Secure decode-and-forward relay SWIPT systems with power splitting schemes. IEEE Trans. Veh. Technol. 2018, 67, 7341-7354. [CrossRef]

35. Zhang, J.; Pan, G. Outage analysis of wireless-powered relaying MIMO systems with non-linear energy harvesters and imperfect CSI. IEEE Access 2016, 4, 7046-7053. [CrossRef]

36. Wang, K.; Li, Y.; Ye, Y.; Zhang, H. Dynamic power splitting schemes for non-linear EH relaying networks: Perfect and imperfect CSI. In Proceedings of the 2017 IEEE 86th Vehicular Technology Conference (VTC-Fall), Toronto, ON, Canada, 24-27 September 2017; pp. 1-5.

37. Zhang, J.; Pan, G.; Xie, Y. Secrecy analysis of wireless-powered multi-antenna relaying system with nonlinear energy harvesters and imperfect CSI. IEEE Trans. Green. Commun. Netw. 2018, 2, 460-470. [CrossRef]

38. Nakagami, M. The $m$-distribution-A general formula of intensity distribution of rapid fading. In Statistical Methods in Radio Wave Propagation; Pergamon Press: New York, NY, USA, 1960; pp. 3-36.

39. Dong, Y.; Hossain, M.J.; Cheng, J. Performance of wireless powered amplify and forward relaying over nakagami- $m$ fading channels with nonlinear energy harvester. IEEE Commun. Lett. 2016, 20, 672-675. [CrossRef]

40. Maleki, M.; Hoseini, A.M.D.; Masjedi, M. Performance analysis of SWIPT relay systems over Nakagami-m fading channels with non-linear energy harvester and hybrid protocol. In Proceedings of the Iranian Conference on Electrical Engineering (ICEE), Mashhad, Iran, 8-10 May 2018; pp. 610-615. 
41. Chen, Y.; Zhao, N.; Alouini, M. Wireless energy harvesting using signals from multiple fading channels. IEEE Trans. Commun. 2017, 65, 5027-5039. [CrossRef]

42. Morsi, R.; Boshkovska, E.; Ramadan, E.; Ng, D.W.K.; Schober, R. On the performance of wireless powered communication with non-linear energy harvesting. In Proceedings of the 2017 IEEE 18th International Workshop on Signal Processing Advances in Wireless Communications (SPAWC), Sapporo, Japan, 3-6 July 2017; pp. 1-5.

43. Chen, X.; Yuen, C.; Zhang, Z. Wireless energy and information transfer tradeoff for limited-feedback multiantenna systems with energy beamforming. IEEE Trans. Veh. Technol. 2014, 63, 407-412. [CrossRef]

44. Gradshteyn, I.S.; Ryzhik, I.M. Table of Integrals, Series, and Products, 7th ed.; Elsevier: Amsterdam, The Netherlands, 2007.

45. Wu, Z.; Cao, J.; Wang, Y.; Wang, Y.; Zhang, L.; Wu, J. hPSD: A hybrid PU-learning-based spammer detection model for product reviews. IEEE Trans. Cybern. 2018, in press. [CrossRef]

46. Zhang, C.; Fan, P.Y.; Xiong, K. Optimal power allocation with delay constraint for signal transmission from moving train to base stations in high-speed railway scenarios. IEEE Trans. Veh. Technol. 2015, 64, 5775-5788. [CrossRef]

(C) 2019 by the authors. Licensee MDPI, Basel, Switzerland. This article is an open access article distributed under the terms and conditions of the Creative Commons Attribution (CC BY) license (http:/ / creativecommons.org/licenses/by/4.0/). 